\title{
Cross-platform comparison of Caenorhabditis elegans tissue extraction strategies for comprehensive
}

\section{metabolome coverage}

Florian M. Geier*,' Elizabeth J. Want, ${ }^{1}$ Armand M. Leroi, ${ }^{2}$ and Jacob G. Bundy ${ }^{1}$

${ }^{1}$ Biomolecular Medicine, Department of Surgery and Cancer, Faculty of Medicine, Sir Alexander

Fleming Building, Imperial College London, South Kensington, SW7 2AZ, U.K. and ${ }^{2}$ Department of

Biology, Faculty of Natural Sciences, Sir Alexander Fleming Building, Imperial College London, South Kensington, SW7 2AZ, U.K.

*Corresponding author.

Email: fg308@imperial.ac.uk

Fax: +44 2075943226 


\section{Abstract}

The nematode Caenorhabditis elegans is widely used as a model organism in many areas of the life sciences. Metabolite profiling (metabolomics/metabonomics) is a powerful means of assigning phenotypes to experimentally perturbed $C$. elegans samples (e.g. mutants, RNAi, or chemical treatments). Tissue extraction is a key step, and high-quality and reproducible extractions are essential to the success of metabolomics studies. We have performed an extensive comparison of different tissue extraction techniques with $C$. elegans, comparing two different solvent systems (chloroform/methanol and aqueous methanol) and six different tissue disruption techniques (including manual grinding in a cooled mortar, homogenization, and various grinding media in both reciprocating and orbital tissue mills). All twelve combinations were then compared by GC-MS, ${ }^{1} \mathrm{H}$ NMR spectroscopy, and UPLCMS, and the results evaluated by both overall multivariate clustering approaches as well as distributions

over individual metabolites/metabolite features of coefficient of variation and yield. The choice of solvent had more influence than the disruption method used, although the homogenizer results were clearly outliers. Overall, we concluded that bead-beating with $80 \%$ methanol solution was a good tradeoff - although it is important to note that the definition of the apparent 'best' method depended on which analytical platform was used to evaluate the results. 


\section{Introduction}

Metabolomics, the untargeted profiling of metabolites, is the most downstream of systems biology omic approaches and aims to identify perturbations in biochemical networks. Compared to gene transcripts and proteins, metabolic networks are much more rapid to respond to perturbations, and metabolomics has been widely used to study responses to external factors, such as toxic insult ${ }^{1}$ or hostmicrobial interactions ${ }^{2}$, giving an integrated picture of an organism's interaction with its environment. It has been successfully employed for clinical, nutritional and epidemiological biomarker research as well for more fundamental scientific studies, such as assigning gene function ${ }^{3,4}$.

The nematode Caenorhabditis elegans is a widely used and extremely well characterised model organism in biological research. In addition to its wide use in developmental biology, genetics, evolutionary biology, and neuroscience, it has become one of the most important model organisms for ageing research ${ }^{5}$. To date, only a small number of studies have applied metabolomic analysis to $C$. elegans $^{6-9}$. However we have no doubt this will increase in the future, as several factors make it highly suited to metabolomics studies. The easy maintenance, suitability for high throughput screens, and short lifecycle are of general value for life sciences research; in addition, the ability to grow it on completely chemically defined medium ${ }^{10}$ and to label with stable isotopes ${ }^{11}$ make it an obvious choice for studying metabolism. Finally, there are extensive research resources, such as thousands of readily-available mutants and GFP constructs, and a whole-genome RNAi construct library ${ }^{12}$, making it an ideal choice for testing hypotheses generated through untargeted profiling.

Metabolomics requires sophisticated chemometric and bioinformatic tools ${ }^{13,}{ }^{14}$, as well as robust analytical platforms, typically nuclear magnetic resonance (NMR) spectrometry or mass spectrometry 
coupled to gas chromatography (GC-MS), capillary electrophoresis (CE-MS) or, more commonly, liquid chromatography (LC-MS). However, because metabolomics aims to cover a vast range of metabolites in complex matrices, it stretches the analytical capabilities in comparison to their classical use for the targeted measurement of a few analytes, A particular problem is that metabolites are highly chemically diverse. Thus, comprehensive coverage requires use of complementary analytical platforms $^{15,16}$ as well as optimized sample preparation protocols ${ }^{17,18}$.

Blaise et. al. ${ }^{19}$ developed a metabolic profiling strategy for $C$. elegans by magic angle spinning (MAS) NMR, which does not require sample extraction and often complements information obtained by conventional NMR metabolite profiling ${ }^{20}$. However, for conventional NMR, and other techniques, such as CE-MS, GC-MS, LC-MS or LC-UV tissue profiling, a prior extraction is needed. C. elegans are both small and tough, with a typical adult size of $1.2 \mathrm{~mm}$ x $60 \mu \mathrm{m}$, and an external cuticle. As a result, tissue extraction protocols designed for work with eukaryotic cells or tissue samples from larger organisms ${ }^{17}$, ${ }^{21,22}$ may not necessarily be effective for C. elegans.

In this study the performance of six different mechanical tissue disruption techniques, each with two extraction solvent systems were evaluated for global untargeted metabolite profiling of C. elegans. For the most comprehensive $C$. elegans metabolome coverage, all samples are profiled and benchmarked by NMR, GC-MS and LC-MS and compared regarding found metabolite features, multivariate clustering, reproducibility and yield. These platforms were selected as GC-MS and reversed phase LC-MS have been shown to be very sensitive and complementary for metabolomics ${ }^{23}$. In addition, NMR is a nearuniversal detector that gives an unbiased and high-precision overview of the most abundant metabolites in a biological sample ${ }^{24}$. 


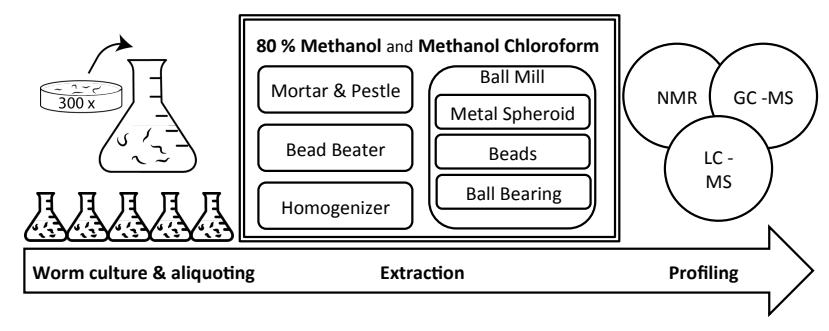

Figure 1. Design of experiment. Three hundred plates with C. elegans were pooled, and divided into 60 aliquots (5 replicates, 6 extraction methods, 2 solvent systems). After extraction, each supernatant was divided for GC-MS, LC-MS and NMR analyses in a ratio of 1:1:8. 


\section{Experimental section}

Materials. All reagents were analytical grade or higher and obtained from Sigma (Dorset, UK). The LC solvents were ultra high purity grade from Romil (Cambridge, UK). C. elegans were obtained from the CGC (Minneapolis, USA).

C. elegans Culture and Sampling. Wildtype N2 C. elegans were grown using standard protocols ${ }^{25}$ at a temperature of $20^{\circ} \mathrm{C}$ on $14 \mathrm{~cm}$ NGM agar plates. We did not synchronize populations, and so the samples analyzed consisted of a mixture of adults and different larval stages. This was in order to give the widest possible metabolome coverage for metabolites that might vary during development, and to test extraction efficiency for worms of different sizes. The worms from 300 healthy plates were washed off with $5 \mathrm{ml} \mathrm{M} 9$ buffer each ${ }^{25}$ and left to settle prior to pooling the obtained pellets. Under constant manual shaking in a conical flask the pooled pellet was partitioned into 60 aliquots in microcentrifuge tubes, each containing $0.5 \mathrm{ml}$. All aliquots were snap frozen in liquid nitrogen and stored at $-80 \mathrm{C}$ until extraction.

Extraction. The overall experimental structure and workflow is summarized in Fig. 1. We compared six different tissue disruption techniques: (1) manual grinding with a $10 \mathrm{~cm}$ ceramic mortar and pestle at liquid nitrogen temperatures, (2) homogenizing with an electric homogenizer (Janke \& Kunkel, Staufen, Germany), (3) bead beating using a FastPrep 12 (MP Biomedicals, Cambridge, UK); and beating in a reciprocating “TissueLyser” ball mill (Qiagen, Crawley, UK) using either (4) metal spheroids, (5) glass beads, or (6) metal ball bearings at cryogenic temperatures. We chose two solvent systems, $80 \% \mathrm{v} / \mathrm{v}$ methanol $(\mathrm{MeOH})$ solution in water, and a monophasic methanol:chloroform:water (MC) modified Bligh and Dyer extraction, which are both used for tissue extraction for metabolite profiling ${ }^{2,21,26}$. In all cases we used a two-step extraction to improve recovery - the first step was direct extraction of the worm pellet, and the second step used an additional volume of solvent for rinsing (mortar and pestle, homogenizer, ball bearing) or re-extraction (bead beater, spheroids, glass beads). For the $80 \%$ methanol extraction, the first step used $2 \mathrm{ml}$ methanol, giving $\sim 80 \%$ methanol in combination with the C. elegans 
worm pellet $(0.5 \mathrm{ml})$. The second step (rinsing/re-extraction) used $1 \mathrm{ml}$ of $80 \%$ methanol. The first step for the MC extraction was $1.2 \mathrm{ml}$ of $2: 1 \mathrm{v} / \mathrm{v}$ methanol:chloroform, to result in a 2:1:1 methanol:chloroform:water mixture with the $400 \mu \mathrm{l}$ pellet. The second step consisted of $0.4 \mathrm{ml}$ chloroform and $0.4 \mathrm{ml}$ water. All extraction solvents used were ice-cold, and the samples were kept on dry ice until immediately before extraction. Detailed protocols, including equipment, volumes and parameters used, can be found in the supporting information (S-1). After extraction, all extracts were centrifuged (5 $\mathrm{min}, 16,000 \mathrm{~g}$ ) and the supernatant topped up to $2 \mathrm{ml}$. After aliquoting for GC-MS, LCMS and NMR in a ratio of 1:1:8, the supernatants were dried overnight in a vacuum sample concentrator at room temperature. All samples were kept at $-80^{\circ} \mathrm{C}$ until analysis. Only the aqueous fraction of the MC extraction was analyzed in this study; a targeted lipidomic protocol for C. elegans has already been developed $^{27}$.

GC-MS Analysis. For GC-MS, we derivatized the samples using the two-step methoximation/silylation protocol of Fiehn et al. ${ }^{28}$. The Fiehn Library ${ }^{28}$ was used for assignment, after deconvolution with AMDIS. Every ten samples an aliquot of a pooled sample was run to serve as quality control (QC) to estimate runtime variance. We also injected this sample five times consecutively in order to assess repeated injection, i.e. machine variance. Detailed experimental procedures can be found in the supporting information S-2.

UPLC-MS Analysis. For LC-MS we used a protocol based on the one recently described by Want et al. ${ }^{29}$ A linear 25 min water to acetonitrile gradient was run on a C18 UPLC column with the coupled TOF-MS detector running in negative mode. The $\mathrm{xcms}$ software package ${ }^{30}$ was used to generate peaklists for further statistical analysis. Exact experimental procedures and acquisition parameters can be found in supporting information S-3. A pooled sample was injected five times prior to the analytical run to condition the column and estimate column conditioning variation. Again, like for GC-MS, the 
pooled sample was injected every ten samples and also five times consecutively to assess runtime and machine variance, respectively.

NMR Analysis. Spectra were acquired using a water-suppressed 1D sequence on a $600 \mathrm{MHz}$ NMR spectrometer, essentially as described by Beckonert et al. ${ }^{31}$ The concentrations of 32 compounds, which we assigned by 2D NMR experiments, were then fitted using NMR Suite 6 (Chenomx, Edmonton, Canada), prior to statistical analysis. Again, full details of the experimental set-up and data processing can be found in the supporting information S-4.

Multivariate Analysis. GC-MS and LC-MS data were started-log transformed ${ }^{32}$ to compensate for the higher dynamic range. For the NMR data, we carried out principal components analysis (PCA) for both the fitted compounds, but also for the full resolution data, following peak alignment ${ }^{33}$. All data were mean centered before performing PCA in R.

\section{Results and Discussion}

In this study we tested six different mechanical tissue disruption methods, each with two solvent systems, for extracting metabolites from C. elegans for GC-MS, LC-MS and NMR profiling. We compared the twelve resulting extraction methods with respect to the number of detected metabolites (NMR, GC-MS) or metabolite features (LC-MS). Overall similarity was judged by clustering, reproducibility, and yield. The raw data for assigned or putatively assigned metabolites (i.e. NMR and GC-MS data) are shown in Fig. 2.

The nematodes grow by feeding on a lawn of bacteria. Although this lawn was largely depleted by feeding at the time of sampling, there would still be bacterial cells present, and so it might be possible 
that this would bias the experiment by contributing to the signal. To test this, we ran a separate experiment comparing extracts of the same volume of worm pellet and supernatant (which should contain only suspended bacteria). This confirmed that the contribution of the bacterial biomass to the overall metabolite profile was negligible (Fig. S-5, online supporting information).

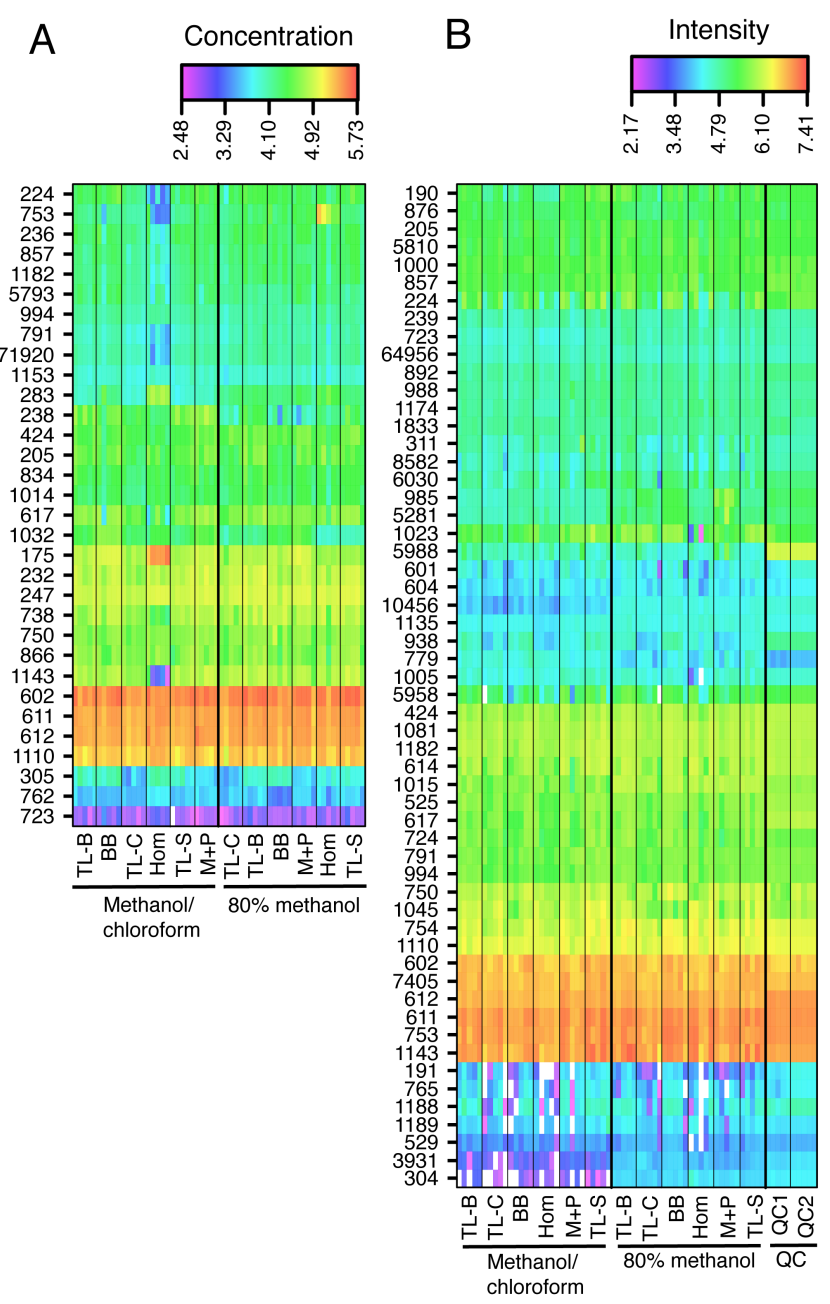

Figure 2. NMR (A) and GC-MS (B) data used for all calculations in this study. Color scale indicates concentration (NMR) or peak integral (GC-MS) on a logarithmic scale. Metabolites are labelled by PubChem ID. TL-B, TL-C, TL-S: TissueLyzer with beads, cryogenic extraction, and spheroids, respectively. BB: bead beating. M+P: mortar and pestle. Hom: rotary homogenizer. QC1: QC samples injected across entire run. QC2: repeated-injection samples. 
${ }^{1}$ H NMR detectable metabolites. We assigned 32 compounds, all of which were confirmed by 2D NMR (table S-7, supplementary information), all of which have been observed in C. elegans before ${ }^{6,7}$. However several metabolites were reported by these earlier studies that we did not observe - e.g. trimethylamine oxide was reported by both Blaise et al. ${ }^{7}$ and Atherton et al. ${ }^{6}$ (although not visible in the HSQC spectrum shown by Atherton et al.), but we did not detect this (nor have we seen this compound in many hundreds of spectra from earlier studies ${ }^{8,9,34}$ ). Kaplan et al. ${ }^{35}$, in contrast, analysed metabolites excreted and/or exuded by C. elegans. It is interesting that many of these were very similar to those found in total tissue extracts, including amino acids and simple carbohydrates (trehalose, glucose, glucose-6-phosphate), although they did also observe metabolites more commonly associated with excretion that we did not detect in total tissue extracts (e.g. allantoin, urea).

We then used commercial software for computer-assisted manual fitting and quantitation of the 32 metabolites in the 1D spectra ${ }^{36}$. GC-MS yielded 54 compounds (supporting information, table S-7) after blank subtraction and manual checking of false positive assignments. This is an increase in the number of metabolites previously reported as detected in C. elegans by GC-MS ${ }^{6,37}$, and our assignment therefore represents important baseline metabolome data. UPLC-MS detected 4903 metabolite features for the $\mathrm{MeOH}$ and 3595 for the $\mathrm{MC}$ extraction across all extraction methods; 5141 features in total across both solvents and all extraction methods. Unambiguous assignment of confirmed metabolites requires high standards for confidence, including matching retention time, isotope and fragmentation pattern of an authentic standard ${ }^{38}$. Given this, and because we detected so many features, we decided not to assign specific metabolites by UPLC-MS: the purpose of this study was not to provide extensive metabolome assignment for $C$. elegans. However, we used the CAMERA software package to remove isotopes and adducts/fragments to estimate the number of possible metabolites ${ }^{39}$. Out of the 5141 
features, it assigned 760 isotopes and 1667 adducts/fragments, leaving 2714 features (53\%) as actual metabolite candidates. Between $50-70 \%$ of all features were either detected in all five or no replicates (Figure S-6, supporting information) of each individual method. Remarkably, features detected in only one, two, three or four replicates of a method often had a coefficient of variation of similar to peaks detected in all (five) replicates of a method. However, inspection of the corresponding extracted ion chromatograms (EICs) showed that that in most of these cases a feature was present in all replicates, but just below the standard detection parameters of the xcms "centWave" peak picker (intensity>100, $\mathrm{SN}>10$ ). Low intensity metabolite features often diminish overall reproducibility for LC-MS data ${ }^{40-42}$. Here, for all further analyses, we decided to include only features detected in all five replicates of at least one method. This is a conservative approach, and means that while there will undoubtedly be some false negatives, we can be confident that we are basing the between-method comparisons on robust data. Even with this stringent criterion, we detected from 1000 to 1366 features per disruption method (Figure S-6, supporting information). In terms of common reproducible features, the mortar and pestle, spheroid, beads (TissueLyser) and bead beater (Fastprep) extraction methods showed the highest overlap within and across solvent systems, with exception of the bead beater in conjunction with MC. The ball-bearing and homogenizer extractions, however, were relative outliers, with the smallest overlap with any of the other methods (Figure S-6, supporting information). It is also interesting to determine which features were exclusive to a single method, i.e. the intersection of features detected in all $(5 / 5)$ and no $(0 / 5)$ replicates of a method. This clearly shows that there are very few features unique to specific tissue disruption methods, but there are clear differences between the two solvents: $\mathrm{MeOH}$ extraction resulted in about 100 to 300 additional reproducible features across all methods compared to MC extraction (Figure S-6, supporting information). These are from lipid metabolites that are solubilized by methanol, but partition into the non-polar chloroform phase during MC extraction. This is shown most clearly by the UPLC chromatograms (Figure S-7, supporting information). 

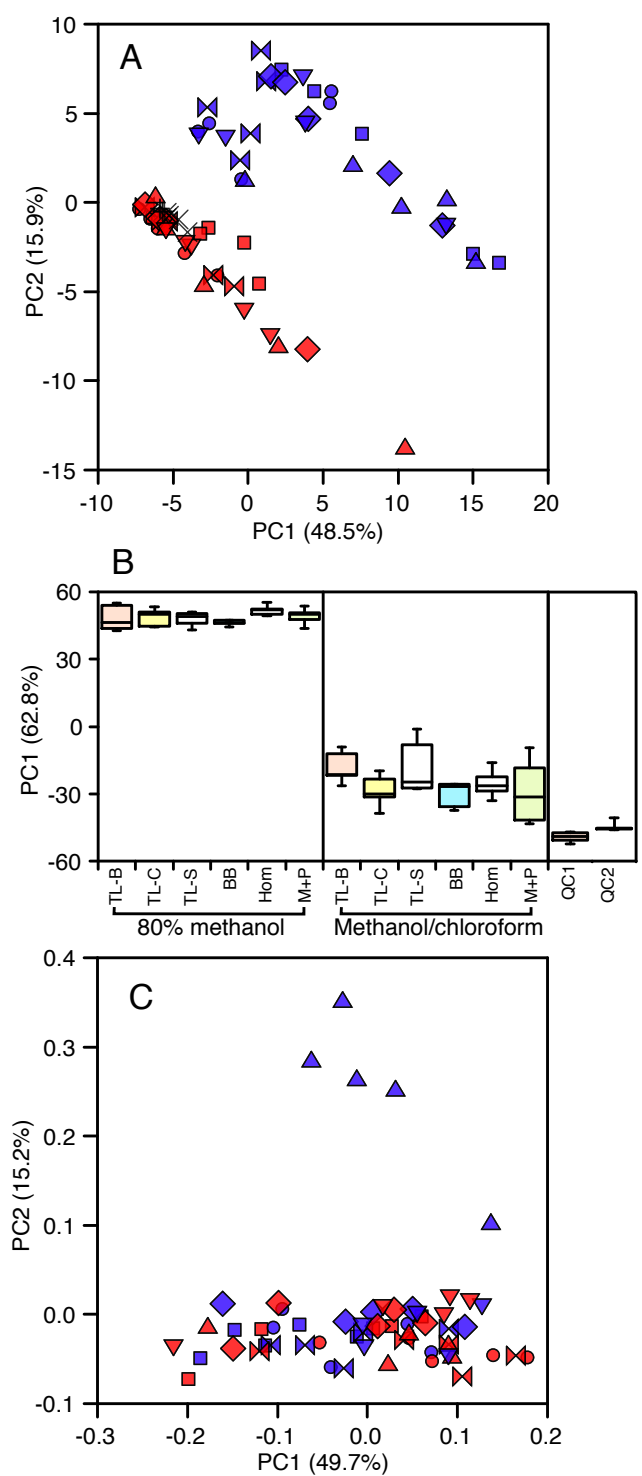

Figure 3. PCA scores plots of the metabolites or metabolite features for GC-MS (A), UPLC-MS (B) and NMR (C), including the percentage variance explained for each principal component (PC). Symbols for $\mathrm{A}$ and $\mathrm{C}:$ blue $=$ methanol/chloroform, red $=80 \%$ methanol. TissueLyser + beads $(\mathrm{TL}-\mathrm{B})$ : circles. TissueLyser + cryogenic grinding (TL-C): squares. TissueLyser + spheroids (TL-S): 'bow-tie' shapes. Bead-beater (BB): diamonds. Homogenizer (Hom): triangles. Mortar and pestle (M+P): inverted triangles. QC samples: black crosses. For the UPLC-MS data (B), only one principal component was fitted, as PC 2 only explained $6 \%$ of the variance, and did not provide any interpretational value. The boxes display the median and first and third quartiles, and the whiskers extend to the extreme data points. QC1; QC samples injected across entire run. QC2: repeated-injection samples. 
Multivariate Clustering. In addition to assessing study precision and yield of individual metabolites/features, we used PCA to provide a simple visualization of the overall effects of the different extraction method/solvent combinations on metabolic profiles. The runtime and (repeated injection) machine variance QC samples for GC-MS and UPLC-MS clustered tightly, indicating robust within-run analytical performance ${ }^{40,41,43}$ (Figure 3). For both mass spectrometric methods there was a clear solvent effect, and for UPLC-MS all the MC samples were more tightly clustered in PC space than the corresponding $\mathrm{MeOH}$ extractions. This wasn't the case for NMR, where neither the aligned fullresolution data (not shown) nor the fitted metabolite concentration data were clearly separated with respect to solvent (Figure 3). This is in agreement with Lin et. al. ${ }^{22}$, who observed that during tissue extraction and ${ }^{1} \mathrm{H}$ NMR profiling, the inter-sample variation was higher than the solvent contribution. In addition, Bundy et al. ${ }^{44}$ found that variation introduced by manual metabolite fitting plus machine reproducibility together was less than the variation between any two biological replicates. However PCA analysis of the 32 fitted NMR compounds clearly showed that the homogenizer/MC extraction samples were distinct from all the others (Figure 3), as can also be seen from the raw data (Figure 2). In particular, trehalose was completely absent, and there was a large increase in acetate. The simplest explanation is that, compared to the other protocols, the homogenizer extraction was the only method where the frozen sample pellet thawed appreciably before the cells were disrupted into the solvent, and therefore there were some biological changes in addition to differences resulting from the extraction method alone. Apart from this, no other method was clearly differentiated in any of the three analytical platforms, indicating that the choice of solvent was much more important than the tissue disruption method in affecting the observed metabolome variance. 

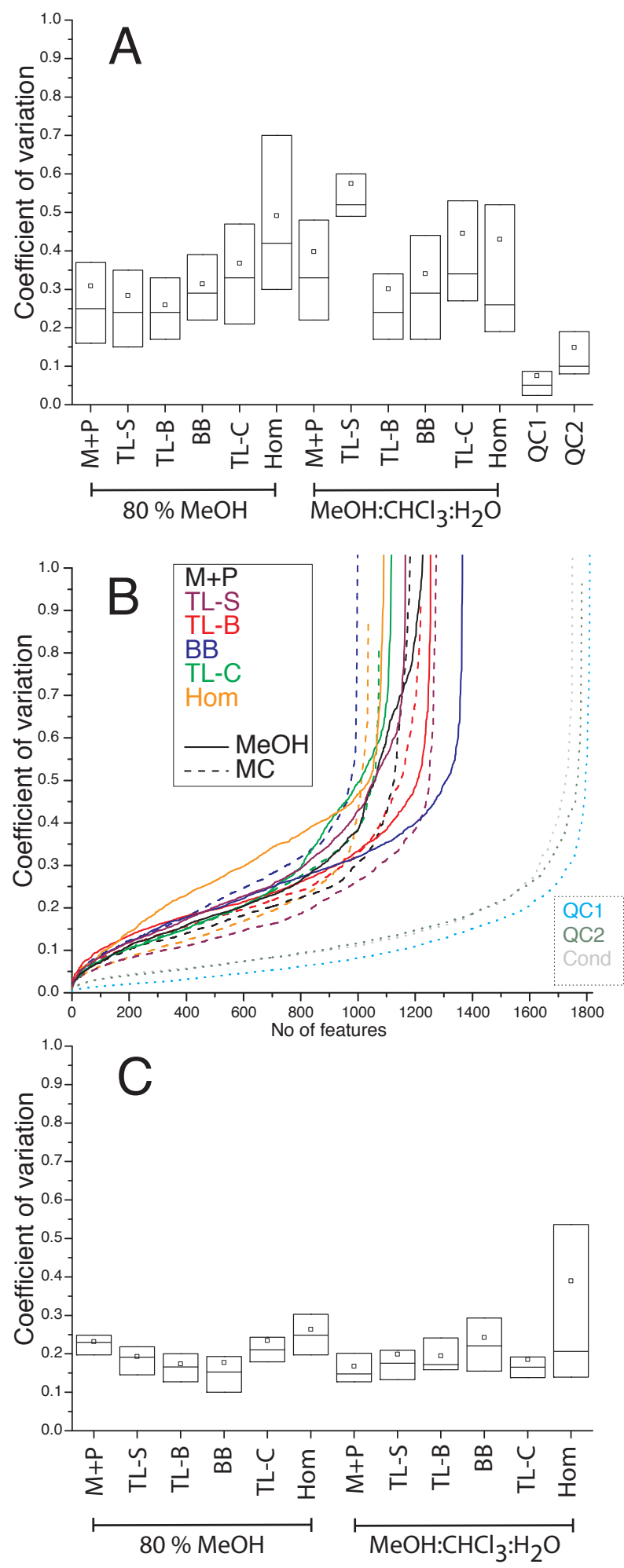

Figure 4. Coefficient of variation (CV) for (a), UPLC-MS (b) and NMR (c) metabolite features. For GC-MS and NMR features the CV distribution is presented as boxplots, whereas for UPLC-MS the CV is ranked in ascending order. The box spans between the $25^{\text {th }}$ and $75^{\text {th }}$ percentile, with a line at median height and a dot representing the mean. TL-B, TL-C, TL-S: TissueLyzer with beads, cryogenic 
extraction, and spheroids, respectively. BB: bead beating. $\mathrm{M}+\mathrm{P}$ : mortar and pestle. Hom: rotary homogenizer. QC1: QC samples injected across entire run. QC2: repeated-injection samples.

Precision. Analytical precision is an important parameter to characterize the reproducibility of an analytical platform and applied protocols. We have chosen to display this across all metabolites using boxplots for the GC-MS (4a) and NMR (4c) data, showing the distribution of coefficients of variation (CV) of the semi-automatically reintegrated and fitted metabolites respectively, as we feel it is important to be able to make visual assessments of data quality, beyond the relative overview of multivariate methods. For the UPLC-MS data, given that we only compared features detected in all $(5 / 5)$ replicates of a method, there were a different number of selected features across the methods. Therefore we chose to present ranked $\mathrm{CV}$ instead, as the boxplots do not account for the different number of variables and so could bias visual interpretation. GC-MS and UPLC-MS both showed a median repeated injection (RI) and quality control sample (QC) variability of $5 \%$ and $10 \%$, respectively. For GC-MS, we could not find any published data on distributions of CV for repeated injection (instrument) variation to compare to, but for LC-MS data our median CV is lower than reported values ${ }^{45,46}$. This might be due to fewer replicates or our minimum quantification criterion $(5$ out of 5 detected features). The median QC CV agrees well with earlier studies. ${ }^{17,40,47}$ The variability of the UPLC-MS “column conditioning” samples (at the beginning of the run) and the QC samples (spaced throughout the run) was nearly identical, with only a few additional features detected exclusively in the QC samples. More interestingly, both the CV of repeated injections and QC samples of the UPLC-MS features rise with the same slope, but with a constant offset, which equals the pure runtime variation (2$3 \%$ ). This constant offset across all features means that in a well performed LC-MS experiment the runtime variation seems to be constant for every feature. The error through pure technical variation (repeated injections), however, appears to depend on the detected metabolite as its CV rises with the 
number of metabolites. The most likely explanation for this observation may be varying ionization reliabilities for different features. Unfortunately, the 54 metabolites found by GC-MS were not sufficient to produce a high resolution CV distribution in order to allow similar observations. For UPLC-MS, xcms detected nearly twice as many features in the QC, conditioning and repeated injection samples compared to the method samples. It is likely that the different methods may to some extent have extracted different sets of metabolites, and so the mixture of all gives a higher number of detected features. This emphasizes that there is not one single extraction strategy that can give a complete picture of the whole metabolome. Generally, nearly all methods investigated showed a median CV of $15-30 \%$ across all platforms. This coincides with values reported for various GC-MS and LC-MS extraction protocols $^{17,18,40,42,47}$. The CVs for the NMR data are relatively high compared to other published studies ${ }^{48-50}$, but appear to be a realistic picture of the combined biological and technical variation in this study. Our previous studies have shown that NMR-derived instrument variation is much less than the variation between two biological replicates, even including the peak fitting procedure ${ }^{44}$. Nonetheless, individual methods (e.g. spheroid/MC extraction for GC-MS; homogenizer/MeOH for UPLC-MS; and homogenizer/MC for NMR) could be identified that clearly were worse performers on the basis of the summary statistics chosen. A similar unexplainable method bias for the same extraction method on a different platform has also been observed by t'Kindt et. $a .^{42}$. The spheroid/MC extracts are particularly intriguing in this respect: for the NMR data, the CV is similar to the other methods, for the GC-MS data this method performs poorly, but it has the lowest CV throughout if judging by the UPLC-MS data.

Yield. It is important to consider yield as well as precision when comparing different protocols. We chose a relative rather than absolute measure to compare between different methods, to avoid difficulties with differing ionization efficiencies for UPLC-MS features. For each metabolite feature, we set the highest intensity for that feature across all methods to $100 \%$ and scaled the others proportionally to achieve a yield distribution for each method (Figure S-9, online supporting information). The median yield for GC-MS ranged from 50\% to nearly $90 \%$, whereas in UPLC-MS the range was from $50 \%$ to 
$75 \%$ and $65 \%$ to $95 \%$ in NMR. Only UPLC-MS showed a clear difference between the two extraction solvents with lower yields for the MC samples, which corresponds with the observation that $\mathrm{MeOH}$ based methods yielded 150 to 300 more features than their MC counterparts. GC-MS and NMR had the highest yield variance between methods, and UPLC-MS within methods. The homogenizer and ballbearing extractions had the lowest yields overall, which might be due to incomplete tissue disruption. In distinction to the comparisons based on precision, there were no cases where a method was found to be simultaneously very good on one platform and very poor on another.

Our results clearly show there is not any one method that provides a comprehensive metabolome snapshot. Most published extraction protocol optimizations almost exclusively focus on solvent systems $17,18,21,22,42,51$, whereas we compared the influence of both different solvents and mechanical tissue disruption methods. Considered across all three analytical platforms, the choice of solvent system $(80 \%$ methanol or chloroform/methanol) clearly had a larger difference than the choice of extraction method. For NMR-based profiling, however, the results for both solvent systems were very similar, and either solvent system could equally well be used. Overall, all of the different extraction techniques gave good results for NMR, except that the use of a rotary homogenizer gave clearly poorer results - for methanol/chloroform in particular, but also for $80 \%$ methanol. However if one had to pick a single method for NMR, the combination of $80 \%$ methanol with a bead-beater gave the most reproducible results overall. For GC-MS, similar to NMR, both solvent systems gave similar results in terms of reproducibility - although the two sets of data for GC were clearly separated by PCA. Again, use of a homogenizer gave relatively poorer results, as did the combination of metal spheroids plus chloroform/methanol. For UPLC-MS, as for GC-MS, there was a much bigger difference between the two solvent systems than between the different extraction methods. In this case, a lot of the difference was down to the fact that the $80 \%$ methanol solution extracts a number of metabolites that partition into the chloroform layer for chloroform/methanol extraction. Hence, the decision as to which solvent system to use for UPLC-MS will partly depend on the study design - e.g. our current study analysed 
only the polar fraction, and it is highly likely that a joint metabolomic/lipidomic analysis of the polar and non-polar fractions would still conclude that chloroform/methanol extraction was preferable.

Thus, based on a comparison of all data, we recommend that rotary homogenizers should not be used. Both $80 \%$ methanol and chloroform/methanol combinations give acceptable results (with very similar distributions of reproducibility) across all three analytical platforms; the main difference is that more lipid peaks are seen in the UPLC-MS. We therefore recommend, for polar metabolome analysis only, use of a bead-beater in combination with $80 \%$ methanol. Use of a bead-beater is recommended because it consistently performed well across all platforms with respect to the total number of metabolites (or metabolite features) detected, precision, and yield; is relatively high-throughput; and minimizes between-person variation. However, if a bead-beater is not available, a mortar and pestle also produced high-quality data. Use of $80 \%$ methanol rather than chloroform/methanol for extraction is recommended because it is simpler (and therefore likely to be more robust for high-throughput studies in particular), and extracts more metabolites (and hence potentially contains more biochemical information) than chloroform/methanol.

\section{Conclusion}

We have conducted a true cross-platform comparison of tissue extraction strategies for untargeted GC-MS, UPLC-MS and NMR metabolite profiling, which shows that individual tissue disruption/solvent system combinations may perform well on one platform but poorly on another. Even though tissue disruption methods largely did not represent the major source of multivariate variation, they showed substantial differences in terms of number of recovered metabolites, precision, and yield, and should therefore be carefully considered for future studies that use tissue extraction. We therefore argue that it is important to validate extraction methods across multiple analytical platforms.

\section{ACKNOWLEDGMENT}


FMG acknowledges the AXA Research Fund and EJW acknowledges Waters Corporation for funding. Kerstin Williams is thanked for access to a "FastPrep" bead-beater. Paul Benton and Sarah Davies are thanked for help with xcms data processing and worm culture, respectively.

\section{SUPPORTING INFORMATION AVAILABLE}

Additional information as noted in text. This material is available free of charge via the Internet at http://pubs.acs.org.

\section{REFERENCES}

(1) Coen, M.; Holmes, E.; Lindon, J. C.; Nicholson, J. K. Chem Res Toxicol. 2008, 21, 9-27.

(2) Swann, J. R.; Want, E. J.; Geier, F. M.; Spagou, K.; Wilson, I. D.; Sidaway, J. E.; Nicholson, J. K.; Holmes, E. Proc Natl Acad Sci U S A. 2010,

(3) Dunn, W. B.; Broadhurst, D. I.; Atherton, H. J.; Goodacre, R.; Griffin, J. L. Chem Soc Rev. 2011, 40, $387-426$.

(4) Holmes, E.; Wilson, I. D.; Nicholson, J. K. Cell. 2008, 134, 714-717.

(5) Collins, J. J.; Huang, C.; Hughes, S.; Kornfeld, K. The measurement and analysis of age-related changes in Caenorhabditis elegans (January 24, 2008) WormBook, ed. The C. elegans Research Community, WormBook, doi/10.1895/wormbook.1.137.1, http://www.wormbook.org.

(6) Atherton, H. J.; Jones, O. A.; Malik, S.; Miska, E. A.; Griffin, J. L. FEBS Lett. 2008, 582, 16611666.

(7) Blaise, B. J.; Giacomotto, J.; Elena, B.; Dumas, M. E.; Toulhoat, P.; Segalat, L.; Emsley, L. Proc Natl Acad Sci U S A. 2007, 104, 19808-19812.

(8) Fuchs, S.; Bundy, J. G.; Davies, S. K.; Viney, J. M.; Swire, J. S.; Leroi, A. M. 2010, 8, 14. 
(9) Swire, J.; Fuchs, S.; Bundy, J. G.; Leroi, A. M. Proc R Soc B. 2009, 276, 2747-2754.

(10) Szewczyk, N. J.; Udranszky, I. A.; Kozak, E.; Sunga, J.; Kim, S. K.; Jacobson, L. A.; Conley, C. A. J Exp Biol. 2006, 209, 4129-4139.

(11) Perez, C. L.; Van Gilst, M. R. Cell Metab. 2008, 8, 266-274.

(12) Kamath, R. S.; Fraser, A. G.; Dong, Y.; Poulin, G.; Durbin, R.; Gotta, M.; Kanapin, A.; Le Bot, N.; Moreno, S.; Sohrmann, M.; Welchman, D. P.; Zipperlen, P.; Ahringer, J. Nature. 2003, 421, 231237.

(13) Ebbels, T. M.; Keun, H. C.; Beckonert, O. P.; Bollard, M. E.; Lindon, J. C.; Holmes, E.; Nicholson, J. K. J Proteome Res. 2007, 6, 4407-4422.

(14) Trygg, J.; Holmes, E.; Lundstedt, T. J Proteome Res. 2007, 6, 469-479.

(15) van der Werf, M. J.; Overkamp, K. M.; Muilwijk, B.; Coulier, L.; Hankemeier, T. Anal Biochem. $\mathbf{2 0 0 7}, 370,17-25$.

(16) Williams, R.; Lenz, E. M.; Wilson, A. J.; Granger, J.; Wilson, I. D.; Major, H.; Stumpf, C.; Plumb, R. Mol Biosyst. 2006, 2, 174-183.

(17) Masson, P.; Alves, A. C.; Ebbels, T. M.; Nicholson, J. K.; Want, E. J. Anal Chem. 2010, 82, 77797786.

(18) Want, E. J.; O'Maille, G.; Smith, C. A.; Brandon, T. R.; Uritboonthai, W.; Qin, C.; Trauger, S. A.; Siuzdak, G. Anal Chem. 2006, 78, 743-752.

(19) Blaise, B. J.; Giacomotto, J.; Triba, M. N.; Toulhoat, P.; Piotto, M.; Emsley, L.; Segalat, L.; Dumas, M. E.; Elena, B. J Proteome Res. 2009, 8, 2542-2550.

(20) Bollard, M. E.; Murray, A. J.; Clarke, K.; Nicholson, J. K.; Griffin, J. L. FEBS Lett. 2003, 553, 7378. 
(21) Le Belle, J. E.; Harris, N. G.; Williams, S. R.; Bhakoo, K. K. NMR Biomed. 2002, 15, 37-44.

(22) Lin, C. Y.; Wu, H.; Tjeerdema, R. S.; Viant, M. R. Metabolomics. 2007, 3, 55-67.

(23) Buscher, J. M.; Czernik, D.; Ewald, J. C.; Sauer, U.; Zamboni, N. Anal Chem. 2009, 81, 21352143.

(24) Nicholson, J. K.; Wilson, I. D. Progr NMR Spectrosc. 1989, 21, 449-501.

(25) Stiernagle, T. Maintenance of C. elegans (February 11, 2006). WormBook, ed. The C. elegans Research Community, WormBook, doi/10.1895/wormbook.1.101.1, http://www.wormbook.org.

(26) Bligh, E. G.; Dyer, W. J. Can J Biochem Physiol. 1959, 37, 911-917.

(27) Matyash, V.; Liebisch, G.; Kurzchalia, T. V.; Shevchenko, A.; Schwudke, D. J Lipid Res. 2008, 49, 1137-1146.

(28) Kind, T.; Wohlgemuth, G.; Lee do, Y.; Lu, Y.; Palazoglu, M.; Shahbaz, S.; Fiehn, O. Anal Chem. 2009, $81,10038-10048$.

(29) Want, E. J.; Wilson, I. D.; Gika, H.; Theodoridis, G.; Plumb, R. S.; Shockcor, J.; Holmes, E.; Nicholson, J. K. Nat Protoc. 2010, 5, 1005-1018.

(30) Smith, C. A.; Want, E. J.; O'Maille, G.; Abagyan, R.; Siuzdak, G. Anal Chem. 2006, 78, 779-787.

(31) Beckonert, O.; Keun, H. C.; Ebbels, T. M.; Bundy, J.; Holmes, E.; Lindon, J. C.; Nicholson, J. K. Nat Protoc. 2007, 2, 2692-2703.

(32) Rocke, D. M.; Durbin, B. Bioinformatics. 2003, 19, 966-972.

(33) Veselkov, K. A.; Lindon, J. C.; Ebbels, T. M.; Crockford, D.; Volynkin, V. V.; Holmes, E.; Davies, D. B.; Nicholson, J. K. Anal Chem. 2009, 81, 56-66. 
(34) Hughes, S. L.; Bundy, J. G.; Want, E. J.; Kille, P.; Sturzenbaum, S. R. J Proteome Res. 2009, 8, 3512-3519.

(35) Kaplan, F.; Badri, D. V.; Zachariah, C.; Ajredini, R.; Sandoval, F. J.; Roje, S.; Levine, L. H.; Zhang, F.; Robinette, S. L.; Alborn, H. T.; Zhao, W.; Stadler, M.; Nimalendran, R.; Dossey, A. T.; Bruschweiler, R.; Vivanco, J. M.; Edison, A. S. J Chem Ecol. 2009, 35, 878-892.

(36) Weljie, A. M.; Newton, J.; Mercier, P.; Carlson, E.; Slupsky, C. M. Anal Chem. 2006, 78, 44304442.

(37) Held, J. M.; White, M. P.; Fisher, A. L.; Gibson, B. W.; Lithgow, G. J.; Gill, M. S. Aging Cell. 2006, 5, 283-291.

(38) Kind, T.; Fiehn, O. BMC Bioinformatics. 2007, 8, 105.

(39) Tautenhahn, R.; Böttcher, C.; Neumann, S. Bioinformatics Research and Development. 2007, 371380.

(40) Gika, H. G.; Macpherson, E.; Theodoridis, G. A.; Wilson, I. D. J Chromatogr B Analyt Technol Biomed Life Sci. 2008, 871, 299-305.

(41) Gika, H. G.; Theodoridis, G. A.; Wingate, J. E.; Wilson, I. D. J Proteome Res. 2007, 6, 3291-3303.

(42) t'Kindt, R.; Morreel, K.; Deforce, D.; Boerjan, W.; Van Bocxlaer, J. J Chromatogr B Analyt Technol Biomed Life Sci. 2009, 877, 3572-3580.

(43) Sangster, T.; Major, H.; Plumb, R.; Wilson, A. J.; Wilson, I. D. Analyst. 2006, 131, 1075-1078.

(44) Bundy, J. G.; Sidhu, J. K.; Rana, F.; Spurgeon, D. J.; Svendsen, C.; Wren, J. F.; Sturzenbaum, S. R.; Morgan, A. J.; Kille, P. BMC Biol. 2008, 6, 25.

(45) Crews, B.; Wikoff, W. R.; Patti, G. J.; Woo, H. K.; Kalisiak, E.; Heideker, J.; Siuzdak, G. Anal Chem. 2009, 81, 8538-8544. 
(46) Zelena, E.; Dunn, W. B.; Broadhurst, D.; Francis-McIntyre, S.; Carroll, K. M.; Begley, P.; O'Hagan, S.; Knowles, J. D.; Halsall, A.; Wilson, I. D.; Kell, D. B. Anal Chem. 2009, 81, 13571364.

(47) Fiehn, O.; Kopka, J.; Dormann, P.; Altmann, T.; Trethewey, R. N.; Willmitzer, L. Nat Biotechnol. 2000, $18,1157-1161$.

(48) Bauer, M.; Bertario, A.; Boccardi, G.; Fontaine, X.; Rao, R.; Verrier, D. J Pharm Biomed Anal. 1998, 17, 419-425.

(49) Keun, H. C.; Ebbels, T. M.; Bollard, M. E.; Beckonert, O.; Antti, H.; Holmes, E.; Lindon, J. C.; Nicholson, J. K. Chem Res Toxicol. 2004, 17, 579-587.

(50) Parsons, H. M.; Ekman, D. R.; Collette, T. W.; Viant, M. R. Analyst. 2009, 134, 478-485.

(51) Wu, H.; Southam, A. D.; Hines, A.; Viant, M. R. Anal Biochem. 2008, 372, 204-212. 
for TOC only

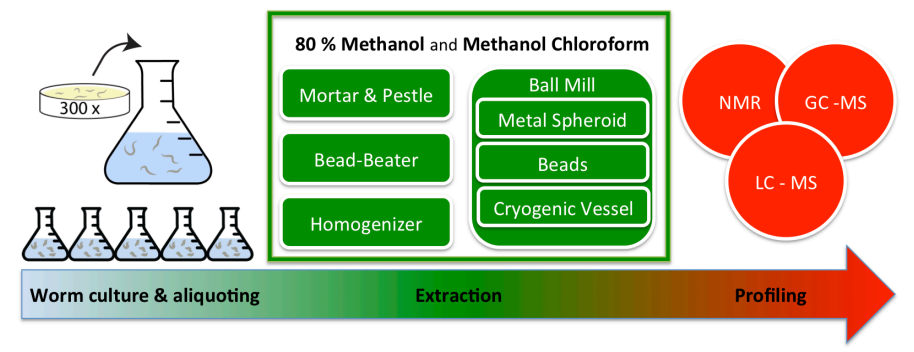

